\title{
Stretchable Kirigami components for composite meso-scale robots*
}

\author{
Amir Firouzeh ${ }^{1,2}$, Tatsuya Higashisaka ${ }^{2,3}$, Keisuke Nagato ${ }^{3}$, Kyujin $\mathrm{Cho}^{1}$, and Jamie Paik ${ }^{2}$
}

\begin{abstract}
Layer-by-layer manufacturing of composite mechanisms allows fast and cost-effective fabrication of customized robots in millimeter and centimeter scales which is promising for research fields that rely on frequent and numerous physical iterations. Due to the limited number of components that can be directly integrated in composite structures, however, often an assembly step is necessary which diminishes the benefits of this manufacturing method. Inspired by the Japanese craft of cutting (kiri-) paper (-gami), Kirigami, we introduce quasi2D and highly stretchable functional Kirigami layers for direct integration into the composite mechanisms. Depending on the material and geometrical design; functional Kirigami layers can perform as flat springs, stretchable electronics, sensors or actuators. These components will facilitate the design and manufacturing of composite robots for different applications. To illustrate the effectiveness of these components, we designed and realized a foldable composite inchworm robot with three Kirigami layers serving as actuator, sensor and contact pad with directional friction. We elaborate on the working principle of each layer and report on their combined performance in the robot.
\end{abstract}

Index Terms-Flexible robots, soft robot materials and design, soft sensors and actuators.

\section{INTRODUCTION}

$\mathbf{M}$ ANUFACTURING robots in millimeter and centimeter scales requires precise assembly of delicate components with miniature features which increases cost and development time. Inspired by the fabrication method of the printed circuit boards, researchers proposed the layer-by-layer manufacturing process to minimize the manual assembly required in the fabrication of complex 3D structures and mechanisms in meso-scale [1], [2]. This manufacturing method has since found application in robotic fields that rely on fast and costeffective iterations for exploring different design principles fields such as educational robotics [3], bioinspired robotics [4], [5], and swarm robotics [6], [7]. However, due to the limited options for quasi-2D functional layers, most mechanisms and robots that are produced within this framework still require an assembly step to integrate functional components such as actuators, sensors, and friction adjusting layers [8]-[13]. In this

Manuscript received: September, 9, 2019; Revised December 9, 2019; Accepted January, 8, 2020.

This paper was recommended for publication by Editor Dezhen Song upon evaluation of the Associate Editor and Reviewers' comments. *This work was supported by the Swiss National Centre for Competence in Research (NCCR) Robotics fund and National Research Foundation of Korea(NRF) Grant funded by the Korean Government(MSIT)(NRF-2016R1A5A1938472).

${ }^{1}$ BioRobotics Lab, Seoul National University, Republic of Korea.

${ }^{2}$ Reconfigurable Robotics Lab, EPFL, Switzerland.

${ }^{3}$ Mechanical Engineering Department, University of Tokyo, Japan.

Digital Object Identifier (DOI): see top of this page.
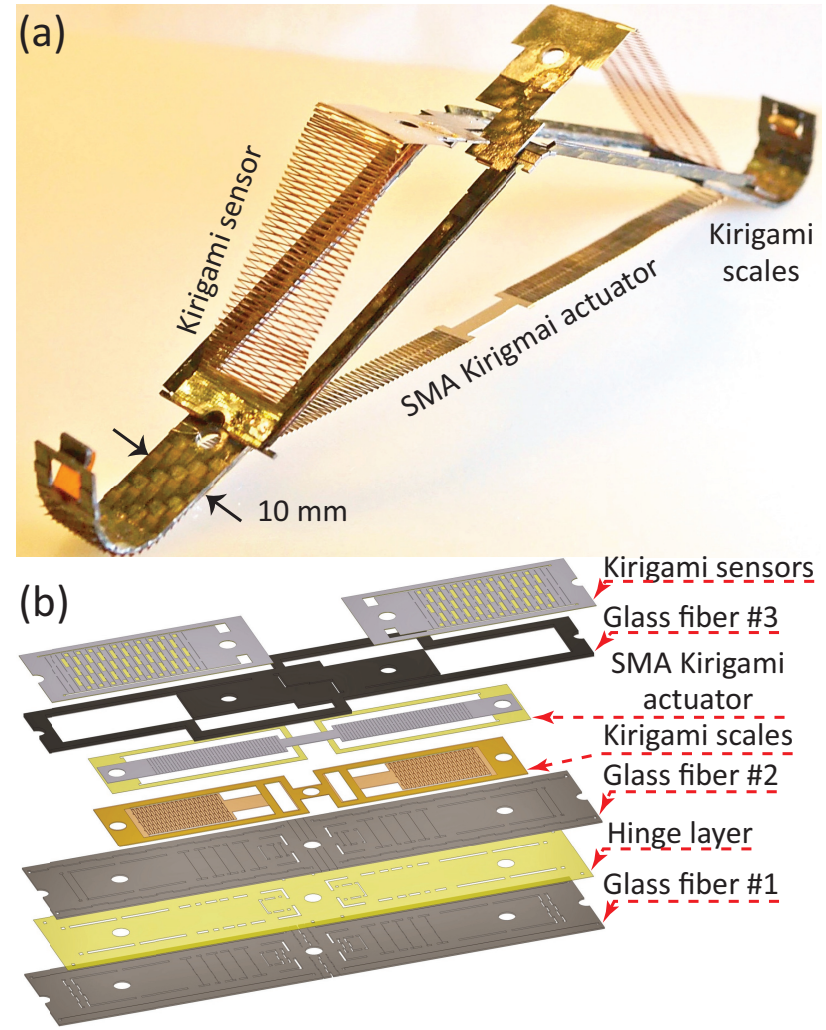

Fig. 1. Integration of functional Kirigami springs in a composite robot. (a) An inchworm composite robot with pop-up Kirigami scales, stretchable Kirigami sensors, and a SMA Kirigami actuator. (b) Construction of the composite inchworm robot.

paper, we introduce quasi-2D stretchable layers with varied functions for easy integration in meso-scale composite robots. Removing the need for post-assembly of such components facilitates the precise and cost-effective mass manufacturing of meso-scale robots with the desired functions integrated.

The functional Kirigami layers that we developed for composite robots are stretchable networks of micro-beams in which the overall elongation is achieved through predominantly bending deformation in the beams. Similar sequence of bending beams have been utilized as springs [14], stretchable electronics [15]-[20], sensors [21]-[23], actuators [24], reflectors with variable angles [25]-[27], and scales [28]. The application of these elements in composite robots, however, have been limited to stretchable electronics [29]. Here, we develop other types of functional Kirigami layers for integration in composite robots.

To design functional Kirigami components, it is necessary to investigate the mechanical behavior of the Kirigami layers. In this paper, we build on previous studies on modeling Kirigami 
springs [14], [30] by considering general material properties (nonlinear elastic properties). This model captures the features of interest in Kirigami springs: the initiation of twist buckling deformation, the twist angle, and the spring stiffness for inplane and out-of-plane deformation modes. We use this model and the scaling laws deduced from it (by considering linear material properties) to design 1- Kirigami pop-up scales for controlling friction properties, 2- Kirigami stretchable sensors, and 3- shape memory alloy (SMA) Kirigami actuators.

To validate the compatibility of the proposed Kirigami components with the layer-by-layer manufacturing process, we integrate these components into a foldable inchworm robot; Fig. 1. This robot is actuated by a SMA Kirigami actuator based on the feedback from two stretchable Kirigami sensors. The pop-up Kirigami scales in this robot determine the direction of motion as the robot moves repeatedly between two configurations. The main contributions of this paper are in:

- Introducing stretchable Kirigami components with varied functions for integration into composite robots: pop-up Kirigami scales, stretchable Kirigami sensors, and shape memory alloy (SMA) Kirigami actuators.

- Modeling the mechanical behavior of Kirigami springs to navigating the design space of functional Kirigami layers.

- Verifying the compatibility of the proposed Kirigami elements with the layer-by-layer manufacturing process for fast and precise fabrication of composite robots in meso-scale.

\section{KIRIGAMI SPRINGS}

The Kirigami pattern utilized throughout this research is composed of a series of parallel cuts as illustrated in Fig. 2a. When stretched, a layer with this pattern behaves as a flat spring. The comprising micro-beams of the Kirigami spring primarily undergo bending deformation as presented in Fig. $2 \mathrm{~b}$. Studying this bending deformation and the overall mechanical behavior of Kirigami springs precede the study of any secondary function of the Kirigami layers. In this section, we study the deformation of Kirigami springs and investigate the mechanical behavior of the sptings with different geometrical design parameters (depicted in Fig. 2a) : length of the cuts $\left(l_{\mathrm{m}}\right)$, gap between the cuts in each column $\left(l_{\mathrm{g}}\right)$, distance between the cuts of successive columns $(b)$, the thickness of the layer $(t)$, and the number of columns and rows $\left(N_{\mathrm{C}}\right.$ and $N_{\mathrm{R}}$ respectively).

When stretched, the micro-beams in the Kirigami springs initially undergo in-plane bending. After a threshold, the beams twist out of plane and start bending about the axis of minimum moment of inertia which requires less energy compared to the in-plane deformation. In a symmetric Kirigami pattern, the twist can happen in any of the two directions at random. To control the twist direction, we designed mechanical "imperfections" on each beam by laser engraving as illustrated in Fig. 2c. These engravings slightly rotate the axis of minimum moment of inertia from the initial horizontal direction. The resulted asymmetry determines the twist direction by making the deformation in one direction less energy demanding; the direction that results in reduced

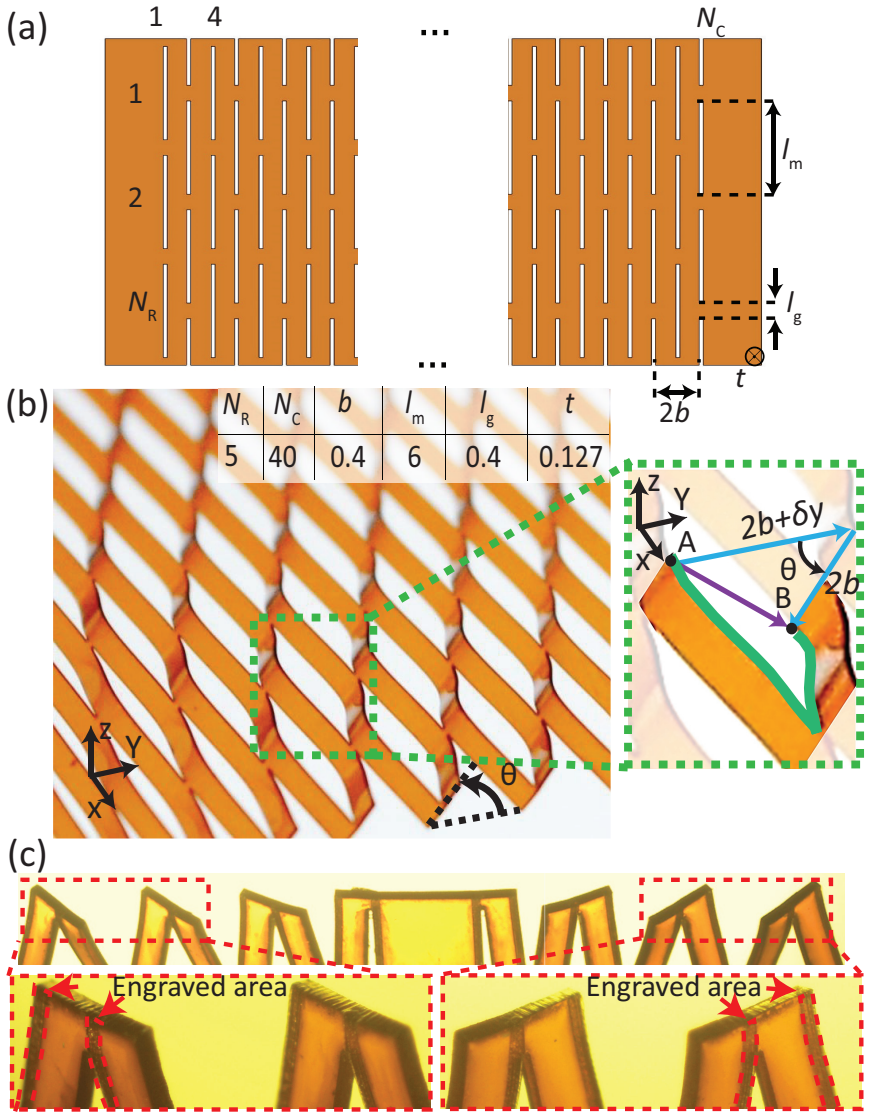

Fig. 2. Kirigami springs. (a) Geometrical design parameters of the kirigami pattern. (b) Deformation of beams in a stretched Kirigami spring and displacement of two initially adjacent points, A and B. Y axis in this figure is along the length of the spring (displacement direction), $\mathrm{X}$ axis is along the width of the spring, and $\mathrm{Z}$ axis is normal to the plane of the spring. (c) A Kirigami spring consisting of two segments with opposing twist directions. The twist direction in each segment is controlled by the laser engraved mechanical imperfections on the surface of the micro-beams.

bending moment of inertia. The opposing twist directions of the beams in the two segments of the Kirigami spring presented in Fig. 2c illustrates the effectiveness of engravings in controlling the twist direction.

The twist angle of the beams in a Kirigami spring increases with stretch; Fig. 3. At any elongation, the twist angle of the beams are the same except for the first few columns. The insets in Fig. 3 show the propagation of the twist angle in the beams. In the first four columns, the twist angle gradually increases. In these beams, the bending and twisting deformations are both prominent. In the rest of the beams, however, the twist angle remains the same and bending is the prominent mode of deformation. For a Kirigami spring with many columns, Forty in our case studies, the mechanical behavior is mainly determined by the dominant bending deformation of the midsection beams. Accordingly, in the analytical model proposed in this research, we neglect the twist buckling deformation of the first few columns and consider solely the constrained bending of the middle section beams.

We use Euler-Bernoulli theory to study the bending deformation of the middle section beams. Given the limitations of the assumptions, the model proposed here is expected to have limited accuracy for large elongation of Kirigami springs with short and wide micro-beams (small $l / b$ ) and for Kirigami 


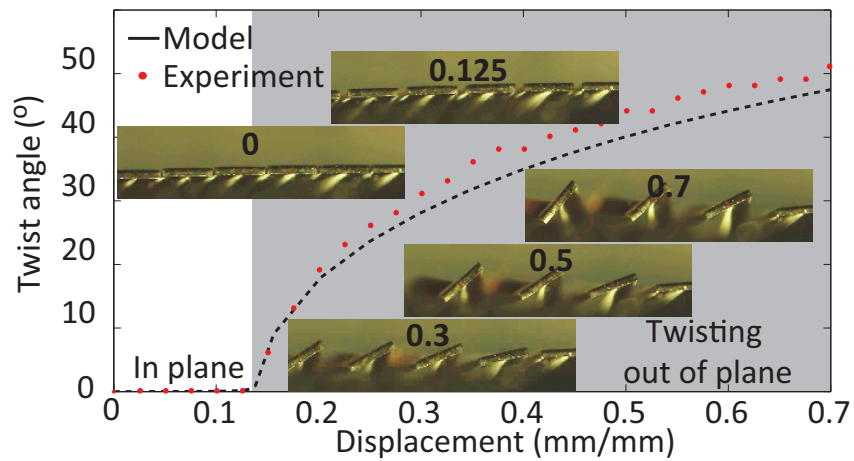

Fig. 3. Twist angle of the micro-beams in a Kirigami spring. After an initial in-plane deformation, the beams twist out-of-plane. The insets show the twist angle propagation in the first few beams. The design parameters of this sample are the same as those of Fig. 2b. The displacement is normalized by the length of the spring $\left(l_{\mathrm{s}}\right)$.

springs with few columns $\left(N_{\mathrm{C}}<40\right)$. The predictions, however, are expected to be accurate for the initial in-plane deformation and for estimating the initiation of the out-of-plane deformation.

The deflection of the beams in Fig. $2 b$ is determined by the imposed boundary conditions on the spring: elongation along the $\mathrm{Y}$ axis and zero displacement along the other two axes. Based on these boundary conditions, the displacement of initially adjacent points A and B in Fig. $2 b$ is calculated as:

$$
\begin{gathered}
D_{\mathrm{y}}=2 b(1-\cos \theta)+2 \frac{\Delta y}{N_{\mathrm{C}}} \\
D_{\mathrm{z}}=-2 b \sin \theta
\end{gathered}
$$

In (1) and (2), $D_{\mathrm{y}}$ and $D_{\mathrm{z}}$ are the displacements along $\mathrm{Y}$ and $\mathrm{Z}$ axes. $\Delta y$ is the overall elongation of the spring, and $\theta$ is the twist angle of the beams. In these equations, we neglected the twist mode of deformation and considered similar bending motion in all of the beams, including the first few columns in which the twist deformation is significant. We also neglected the twist deformation in each beam. To account for these neglected deformation modes and other effects such as the laser beam spot size (which makes the actual dimensions smaller than the designed values), we introduced two correction factors. The product of the first correction factor $\left(k_{1}\right)$ and the beam width $(b)$ yields the effective beam width, $b_{\mathrm{e}}$. The second correction factor is used in evaluating the effective length of the beam; $l_{\mathrm{e}} . l_{\mathrm{e}}$ is defined as the sum of the nominal length $\left(l_{\mathrm{m}}-l_{\mathrm{g}}\right)$ and $k_{2}$ portion of the beam's width. For different materials and within a desired range of design parameters, these corrections should be applied to calibrate the model based on characterization experiments on a few springs with different geometrical design parameters.

The displacement of point B, relative to point A in Fig. 2 is calculated by integrating the radii of curvatures about $\mathrm{Y}$ and $\mathrm{Z}$ axes, $\rho_{\mathrm{y}}$ and $\rho_{\mathrm{z}}$, along the two bending beams connecting points $\mathrm{A}$ and $\mathrm{B}$; highlighted in Fig. $2 \mathrm{~b}$.

$$
\begin{aligned}
& D_{\mathrm{y}}=2 \int_{0}^{l_{\mathrm{e}} / 2} \int_{0}^{l_{\mathrm{e}} / 2} \frac{1}{\rho_{\mathrm{z}}(x)} \mathrm{d} x^{2} \\
& D_{\mathrm{z}}=2 \int_{0}^{l_{\mathrm{e}} / 2} \int_{0}^{l_{\mathrm{e}} / 2} \frac{1}{\rho_{\mathrm{y}}(x)} \mathrm{d} x^{2}
\end{aligned}
$$

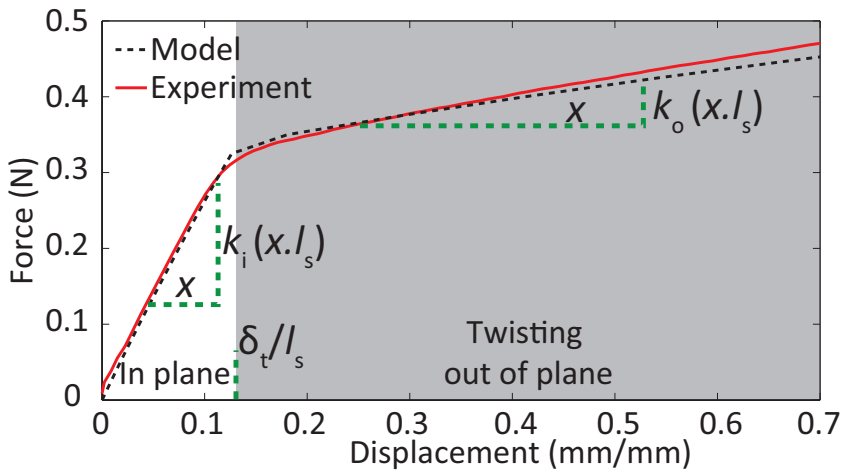

Fig. 4. Mechanical behavior of a Kirigami spring is characterized by two spring constants $K_{\mathrm{i}}$ and $K_{\mathrm{o}}$ (corresponding to in-plane and out-of-plane modes of deformation) and the transition displacement between the two modes $\left(\delta_{t}\right)$. The design parameters of this sample are the same as those of Fig. 2b. The displacement is normalized by the length of the spring $\left(l_{\mathrm{s}}\right)$.

The radii of curvatures at each cross-section of the beam are evaluated by solving the following equations for the bending moments $M_{\mathrm{y}}$, and $M_{\mathrm{z}}$ :

$$
\begin{gathered}
M_{\mathrm{y}}=\iint z \sigma\left(\varepsilon_{\mathrm{x}}\right) \mathrm{d} A, M_{\mathrm{y}}=0 \\
M_{\mathrm{z}}=-\iint y \sigma\left(\varepsilon_{\mathrm{x}}\right) \mathrm{d} A, M_{\mathrm{z}}=\frac{F}{2 N_{\mathrm{R}}} x
\end{gathered}
$$

To integrate over the beam's cross-section in (5) and (6), we use the effective beam width $\left(b_{\mathrm{e}}\right)$ instead of the design value (b) as discussed earlier. $\sigma\left(\varepsilon_{\mathrm{x}}\right)$ in these equations evaluates stress, $\sigma$, as a function of strain, $\varepsilon_{\mathrm{x}}$. For polyimide springs, we used the material characterization results in evaluating this function and accounted for material's nonlinear behavior. Considering the assumptions for the Euler-Bernoulli beam, the strain at different points in each cross-section is evaluated as:

$$
\varepsilon_{\mathrm{x}}=\frac{z^{2}}{\rho_{\mathrm{y}}}-\frac{y z}{\rho_{\mathrm{z}}}
$$

Equations (1)-(7) can be solved numerically for a given spring elongation, $\Delta y$, to determine the corresponding twist angle of the beams and the required tensile force. After a certain threshold, determined by the shape of the cross-section and material properties, the proposed set of equations has one solution for in-plane deformation and an additional solution for out-of-plane deformation; with the out-of-plane solution being significantly less energy demanding and physically valid. Fig. 3 presents the twist angle of the middle section beams as a function of spring elongation. The mechanical behavior of the Kirigami spring changes significantly when the spring is stretched beyond the transition displacement $\left(\delta_{\mathrm{t}}\right)$ as presented in Fig. 4; tenfold drop in the spring constant for the case presented in this figure. The model predictions show good compatibility with the experiment results for the twist angle of the beams, Fig. 3, and for the output force from the spring; Fig. 4.

For polyimide springs that are studied here, the correction factors in the model were determined using the results of experiments on nine samples; $k_{1}=0.95$ and $k_{2}=1.4$. Table I summarizes the design parameters and mechanical behavior of calibration samples. The effect of the design parameters 
TABLE I

PARAMETRIC STUDY OF MECHANICAL BEHAVIOR OF POLYIMIDE KIRIGAMI SPRINGS. ALL SPRINGS HAVE 5 ROWS AND 40 COLUMNS.

\begin{tabular}{|c|c|c|c||c|c|c|}
\hline \multicolumn{9}{|c||}{ Design parameters $(\mathrm{mm})$} & \multicolumn{3}{c|}{ Characteristics (model / experiment) } \\
\hline$l_{\mathrm{m}}$ & $l_{\mathrm{g}}$ & $b$ & $t$ & $k_{\mathrm{i}}(\mathrm{N} / \mathrm{m})$ & $\delta_{\mathrm{t}}(\mathrm{mm})$ & $k_{\mathrm{o}}(\mathrm{N} / \mathrm{m})$ \\
\hline 4 & 0.4 & 0.4 & 0.127 & $447 / 483$ & $2.2 / 2.1$ & $14.2 / 23.7$ \\
6 & 0.4 & 0.4 & 0.127 & $142 / 152$ & $2.3 / 2.2$ & $11.7 / 13.6$ \\
8 & 0.4 & 0.4 & 0.127 & $63 / 69$ & $2.2 / 2.0$ & $6.7 / 7.6$ \\
8 & 0.8 & 0.4 & 0.127 & $72 / 82$ & $2.3 / 2.0$ & $7.3 / 8.3$ \\
8 & 1.2 & 0.4 & 0.127 & $82 / 88$ & $2.3 / 2.1$ & $8.6 / 9.0$ \\
\hline 8 & 0.4 & 0.4 & 0.127 & $63 / 69$ & $2.2 / 2.0$ & $6.7 / 7.6$ \\
8 & 0.4 & 0.6 & 0.127 & $215 / 220$ & $1.3 / 1.4$ & $8.1 / 10.6$ \\
8 & 0.4 & 0.8 & 0.127 & $450 / 474$ & $1.1 / 1.1$ & $9.3 / 12.4$ \\
\hline 4 & 0.4 & 0.4 & 0.051 & $203 / 173$ & $0.3 / 0.4$ & $2.8 / 4.7$ \\
4 & 0.4 & 0.4 & 0.076 & $415 / 377$ & $0.7 / 0.8$ & $3.8 / 12$ \\
4 & 0.4 & 0.4 & 0.127 & $447 / 483$ & $2.2 / 2.1$ & $14.2 / 23.7$ \\
\hline \multicolumn{8}{|c|}{ Kirigami spring design (model / verification) } \\
\hline 4.8 & 0.4 & 0.45 & 0.127 & $385 / 378$ & $1.9 / 2$ & $-/ 22$ \\
\hline
\end{tabular}

in this table are studied in three groups; the effect of the length $\left(l_{\mathrm{m}}-l_{\mathrm{g}}\right)$, width $(b)$, and thickness, $(t)$ of beams. The first block in this table shows that the transition displacement $\left(\delta_{\mathrm{t}}\right)$ is independent of the length of the beams $\left(l_{\text {mesh }}-l_{\text {gap }}\right)$. The spring constant for in-plane and out-of-plane portions $\left(k_{\mathrm{i}}\right.$ and $k_{\mathrm{o}}$ ), however, decrease sharply as the beams get longer (considering linear elastic material $k_{\mathrm{i}}, k_{\mathrm{o}} \alpha l_{\mathrm{e}}{ }^{3}$ ). The transition displacement is determined by the cross-section of the beams. Thin and wide beams transition to out-of-plane mode of deformation at smaller elongation (considering linear elastic material $\left.\delta_{\mathrm{t}} \alpha t^{2} / b\right)$. Changing the cross-section of the beams also affects the spring constants (considering linear elastic material $\left.k_{\mathrm{i}} \alpha b^{3} t, k_{\mathrm{o}} \alpha t^{3} b\right)$.

As demonstrated in Fig. 3, Fig. 4 and Table I, the model captures the important aspects of the mechanical behavior of the Kirigami springs. We verified the applicability of the model by using it to design a Kirigami spring with a desired transition point $\left(\delta_{\mathrm{t}}, k_{\mathrm{i}}\right)$. The last row in Table I presents the parameters of this spring and compares its transition point with the desired one. This model can be used for designing functional Kirigami layers from different materials; given the availability of the mechanical properties $\left(\sigma\left(\varepsilon_{\mathrm{x}}\right)\right)$ and correction factors $\left(k_{1}, k_{2}\right)$. Detailed characterization of material properties and performing parametric studies for all functional Kirigami layers are out of the scope of this paper. So, in the design of the functional layers in the next Section, we use the scaling laws that assumes linear elastic behavior for the material. Table I shows that the nonlinear material properties has a significant effect on the behavior of the springs and the scaling laws can not capture the effect of change in different design parameters as well as the model. The scaling laws, however, can capture the overall trends and by using them we were able to realize return springs (sensors) and a SMA actuator for the inchworm robot with desired behavior within a few physical iterations.

We should point out that while the model provides good predictions for the first loading cycle, the consequent loading cycles can be different depending on the plastic deformation of the material and creep in the polymer. The amount of plastic deformation depends on the design parameters, the material properties and the loading range of the spring. In this paper, we experimentally verified the performance of the functional Kirigami layers in few cycles. Detailed study of the elastic range of deformation of Kirigami springs and including the effects of plastic deformation in the model will be a topic for future studies.

\section{FUNCTIONAL KIRIGAMI LAYERS}

In Section II, we studied the deformation in the microbeams of the Kirigami springs and proposed a model that correlates the geometrical design parameters and the mechanical behavior of the spring. The choice of materials is another important design parameter in defining the function of a Kirigami spring. Here, we use materials with particular mechanical and electrical properties to develop Kirigami springs with different functions: Kirigami scales to produce directional friction, Kirigami sensors, and Kirigami actuators.

\section{A. Pop-up Kirigami scales}

The twist buckling of the beams in a Kirigami spring creates complex 3D structures that are difficult to produce using traditional manufacturing techniques. We use this feature in the pop-up Kirigami scales to create desired textures on the surface of composite robots for producing directional friction. The pop-up Kirigami scales will be integrated into composite robots in their initial quasi-2D state and are deployed to their final 3D shape by elongation. The model predictions can be used to determine the geometrical parameters and the elongation value to achieve a desired twist angle in the deployed state.

Fig. 5a presents the deployment of Kirigami scales and the principle of producing directional friction. Moving in the principle direction of friction, direction 1, results in increased twist angle for the engaged scales, which in turn lifts the robot and increases the contact force and the resisting force at the point of engagement. Moving in the opposite direction, however, results in decreasing the twist angle, which in turn allows the engaged scales to pass over the roughness. To control the twist direction, which determines the principal direction of friction, we engraved the surface of the beams; as discussed in Section II. The triangular patterns in the scales

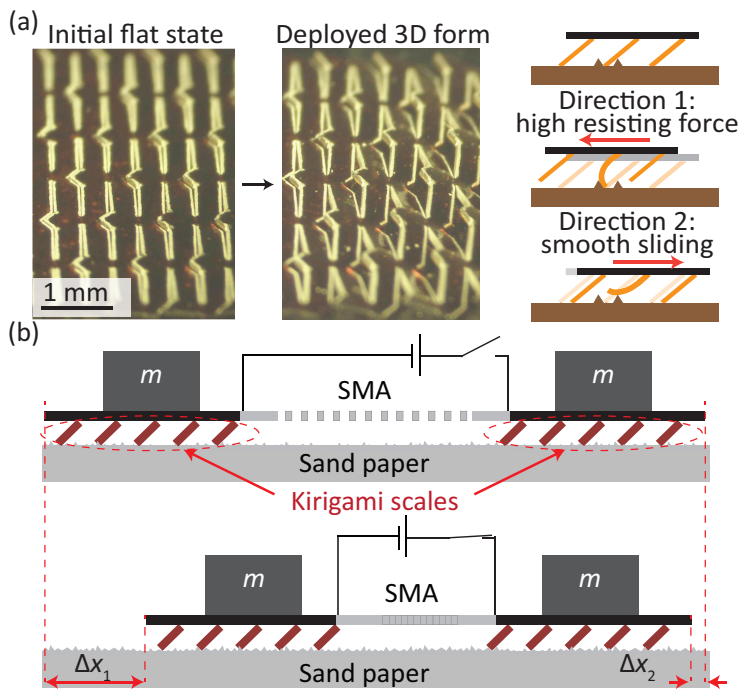

Fig. 5. Kirigami pop-up scales. (a) The out-of-plane deformation of the beams produce complex 3D structures that determines the friction properties. (b) Experiment setup to characterize the performance of Kirigmai scales. 
TABLE II

PARAMETRIC STUDY OF THE PREFORMANCE OF KIRIGAMI SCALES.

\begin{tabular}{|c|c|c|c|}
\hline$\theta\left(^{\circ}\right)$ & $m$ (gr) & Roughness (grit) & $\Delta x_{1} /\left(\Delta x_{1}+\Delta x_{2}\right)^{*}$ \\
\hline 17 & 1 & 320 & mean $=0.95, \mathrm{SD}=0.04$ \\
22 & 1 & 320 & mean $=0.92, \mathrm{SD}=0.05$ \\
41 & 1 & 320 & mean $=0.87, \mathrm{SD}=0.11$ \\
\hline 41 & 2.1 & 320 & mean $=0.86, \mathrm{SD}=0.15$ \\
41 & 5.3 & 320 & mean $=0.80, \mathrm{SD}=0.11$ \\
41 & 10.5 & 320 & mean=0.76, SD $=0.12$ \\
\hline 41 & 5.3 & 320 & mean $=0.80, \mathrm{SD}=0.11$ \\
41 & 5.3 & 240 & mean $=0.86, \mathrm{SD}=0.08$ \\
41 & 5.3 & 100 & mean $=0.99, \mathrm{SD}=0.01$ \\
\hline
\end{tabular}

Mean and standard deviation are for the best 7 of 10 repetitions.

are added to the original Kirigami design to concentrate the contact forces and promote mechanical interference with the substrate.

To study the effectiveness of the Kirigami scales in producing directional friction, we tested pop-up scales in different conditions. We studied the effect of different twist angles $\left(17^{\circ}\right.$, $22^{\circ}$, and $41^{\circ}$ ), loading (in the range of 1-10.5 gr) and surface roughness using different grades of sand paper (100, 240, 320 grit). Fig. 5b presents the schematic of characterization experiments and Table II summarizes the results. In general, scales with smaller twist angle showed slightly better performance, increasing the weight diminished the effectiveness of the scales (due to the deformation of the twisted beams), and the scales had better performance on rougher surfaces. While more detailed study on deformation of Kirigami scales under contact forces is required for optimizing the design of this layer, all the cases studied within this work have shown clear tendency in producing motion in the desired direction.

After integration as a flat sheet into a composite structure, Kirigami scales can be deployed by stretching either during the folding process or by using an integrated actuator. The second method allows for adjustments in the twist angle (or twist direction) and friction properties depending on the task and surface conditions. Ideally, by using active materials, a popup scale layer can take on the task of actuation on its own. To demonstrate this possibility, we designed and manufactured a SMA scale layer with asymmetric engravings capable of changing the twist direction of the scales (presented in the supplementary video). In the application proposed here (Fig. 1), however, we used passive polyimide scales. In the future, active scales will replace the passive ones for changing robot's direction of motion on demand.

\section{B. Stretchable Kirigami sensors}

Kirigami springs transform large displacements at their two ends into bending deformation of their micro-beams. This transformation has been used to manufacture highly stretchable electronics with desired mechanical and electrical properties from composites of metallic foils and polymer layers. The study of the bending deformation of the beams and the alternating strain distribution enables us to design electrically conductive Kirigami layers with sensitive electrical resistance to elongation. These thin Kirigami layers can easily be embeddable into composite foldable robots and provide the required feedback to control their configurations.

The out-of-plane bending deformation of micro-beams in the Kirigami layers takes place about the axis of minimum

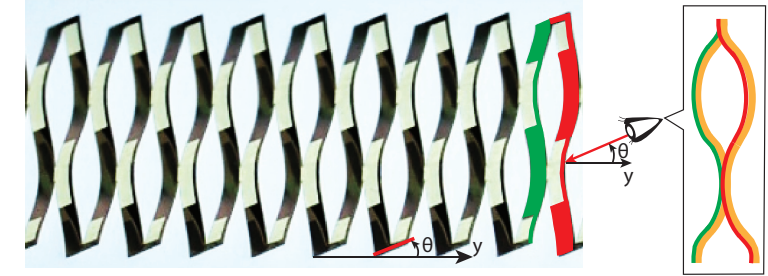

Fig. 6. Deformation of a Kirigami sensor. The electrically conductive metal path (the darker parts) is in tension along half of its length and in compression for the other half. The metal path in two columns are highlighted with different colors. The side view of the sensor shows the alternating curvature direction that results in alternating strain in the metal layer. In this sensor, the higher electrical resistance of the half in tension (narrow sections), results in overall resistance increase with elongation.

moment of inertia; as depicted in the side view in Fig. 6. This puts the material at each cross-section of the beam partly in tension and partly in compression. In the Kirigami sensors, the thicknesses of the polymer layer $(50 \mu \mathrm{m}$ polyimide sheet and $25 \mu \mathrm{m}$ adhesive film) and metal foil (10 $\mu \mathrm{m}$ Constantan) are designed in order to place the neutral plane in the polymer layer and close to the intersection with the metal layer. As a result, the metal layer at each cross-section would be entirely in tension or compression as the beam bends about the axis of minimum moment of inertia. The alternating bending direction of the beams places the metal in tension for half of the length of the beams and in compression in the other half. In a symmetric design, the alternating sign of the normal strain in the metal traces results in no overall sensitivity to elongation. By modulating the width of the metal layer (as highlighted in Fig. 6); via combination of laser engraving and chemical etching; we can increase the electrical resistance in either the sections with metal layer in tension or compression. This increases the contribution of that segment to the overall electrical resistance and hence the overall electrical resistance change. Accordingly, we can design sensors with either positive or negative sensitivity to elongation.

The mechanical behavior of Kirigami sensors matches the behavior of Kirigami springs; discussed in section II. Fig. 7 presents the first and the tenth loading cycles of the Kirigami sensor and reports on the plastic deformation resulted from each cycle. The first cycle clearly shows the two distinct regions of in-plane and out-of-plane bending. The residual strain in the repeated cycles (that largely happens in the first three cycles) smoothens the behavior as seen in the tenth

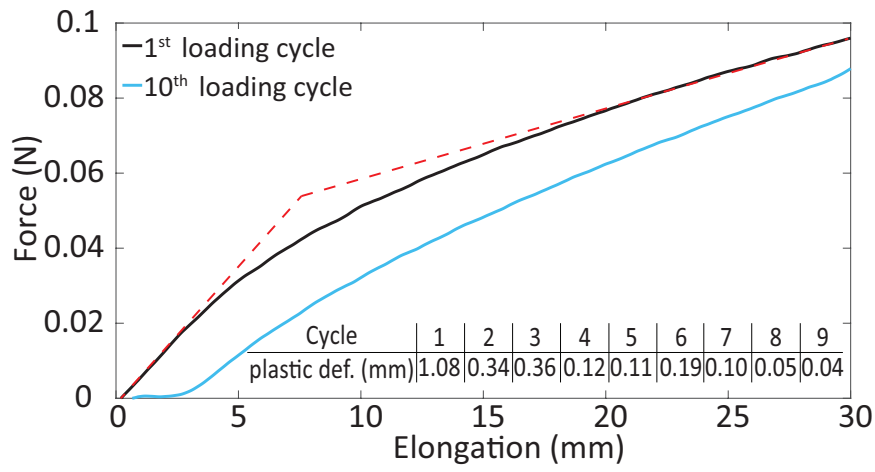

Fig. 7. Mechanical behavior of the Kirigami sensor in the first and the tenth loading cycles. The Plastic deformation mainly occurs in the first few cycles. The trend lines are added to emphasize the dual mechanical behavior of the sensor for in-plane and out-of-plane modes of deformation. 


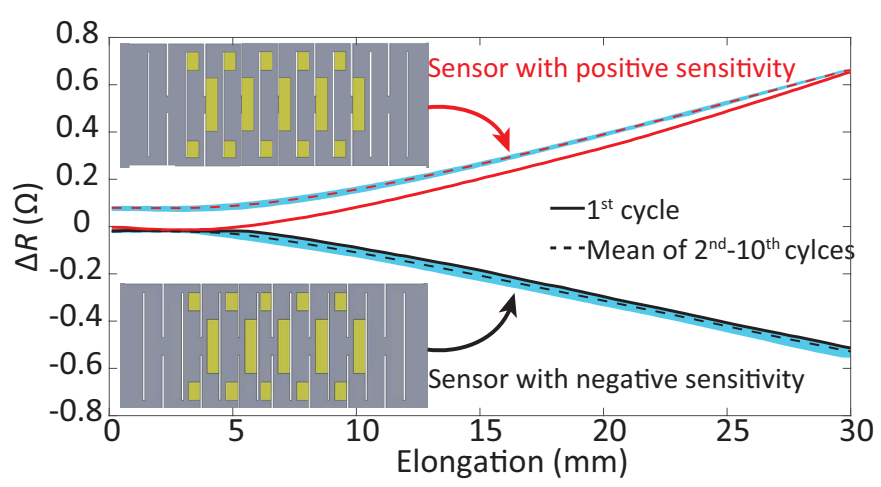

Fig. 8. Electrical resistance variation of the Kirigami sensors in 10 loading cycles. The in-plane deformation of the sensors results in the initial insensitive portion. The plastic deformation in the first cycle results in drift in measurements of one of the sensors. The readings in the consecutive cycles are coherent and reliable; measurements of the $2^{\text {nd }}-10^{\text {th }}$ cycles fall in the shaded area.

loading cycle. While the plastic deformations reported in this figure are not detrimental to sensor's role as a spring, it is necessary to examine the electrical resistance of the sensor and verify the reliability of its measurements.

Fig. 8 presents the readings of two sensors with opposite sensitivities in their first 10 loading cycles. Similar to the imperfections in Fig. 2c, the location of the etching pattern in a Kirigami sensor determines the twist direction of the beams and the loading condition of the segments with narrow metal path (Fig. 6). This in turn determines sensitivity of the sensor (metal layer in narrow sections in tension results in positive sensitivity and in compression results in negative sensitivity). Both sensors in Fig. 8 show fairly linear sensitivity to elongation for the entire range except for the first part that correspond to in-plane mode of deformation (in accordance with mechanical behavior of the sensors presented in Fig. 7). As expected plastic deformation in the first loading cycle results in a drift in the sensor measurements (we suspect that stretching in the manufacturing process has already preloaded the sensor that does not show any drift). The measurements for the following cycles, however, are coherent for both sensors. More details about the design, manufacturing, and modeling of these sensors are presented in our earlier publications on Kirigami sensors [22], [23].

Using Kirigami sensors with opposite sensitivities in parallel allows us to account for the effects of the environmental conditions (e.g. temperature) on the measurements. This is specifically of interests in composite robots that use heat activated actuators. In the SMA actuated inchworm robot (Fig. $1)$, we integrated two Kirigami sensors with similar mechanical properties, overall electrical resistance (around $800 \Omega$ ), and loading condition but opposite sensitivity. The combined measurement of these sensors provides reliable feedback of the state of the robot.

\section{SMA Kirigami actuator}

SMA actuators are effective solutions for driving mesoscale robots because of their high energy density and minimum required auxiliary equipment. However, the conventional forms of SMA actuators, i.e. wires and coil springs, are not well adapted for integration into composite robots. So in most cases

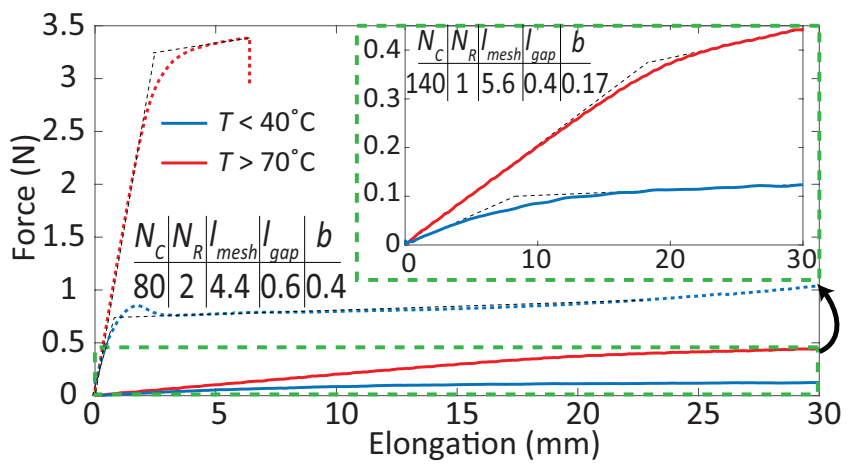

Fig. 9. Mechanical behavior of SMA Kirigami actuators in temperatures above and below SMA transition temperature $\left(55^{\circ} \mathrm{C}\right)$. By altering the design parameters following the scaling laws, presented in Section II, springs with desired loading characteristics are developed.The trend lines are added to emphasize the dual mechanical behavior of the actuator for in-plane and outof-plane modes of deformation.

SMA actuators are assembled onto the composite robots at a separate step. To overcome this limitation, we introduce quasi-2D SMA Kirigami actuators that are compatible with the layer-by-layer manufacturing of the composite robots. Fig. 9 presents the temperature dependent behavior of two SMA Kirigami springs. Around the Austenite transition temperature $\left(55^{\circ} \mathrm{C}\right)$, the spring constant varies more than three times which can be used to actuate composite robots. Given the large recoverable strain of material, plastic deformation in SMA actuators in repeated cycles was negligible (while the actuator was loaded within the range presented in Fig. 9 for hot and cold states).

Material non-linearity has significant effect on the behavior of SMA actuators. Instabilities in the behavior of the harder spring in Fig. 9 and different transition points for the same spring in the hot and cold states (extended in-plane deformation for the hot state) are clear results of material's nonlinear behavior. The mechanical model of section II that considers material nonlinearity can be applied to SMA kirigami actuators. This, however, requires SMA material characterization and calibration experiments on SMA springs which is out of the scope of this paper. In this work, we used the scaling laws (which assume linear elastic material properties) as our guide to navigate the design space of SMA actuators. While these scaling laws can not be used to directly design a SMA actuator, we used them to realize an actuator with desired mechanical behavior for the inchworm robot with a few iterations.

Direct-drivability (through Joule heating), fast cooling (due to large surface area) and compatibility with the laser micromachining manufacturing technique make SMA Kirigami actuators an attractive solution for meso-scale composite robots. Moreover, the multiple design parameters of the Kirigami actuators provides a design space as extensive as that of the coil SMA actuators (reflected in the examples of Fig. 9) allowing their adoption in different applications.

\section{FOLDABLE INCHWORM ROBOT WITH FUNCTIONAL KIRIGAMI LAYERS}

Compatibility with the layer-by-layer manufacturing process, allows easy integration of multiple functional Kirigami layers into foldable composite robots. In this Section, we study 
(a)

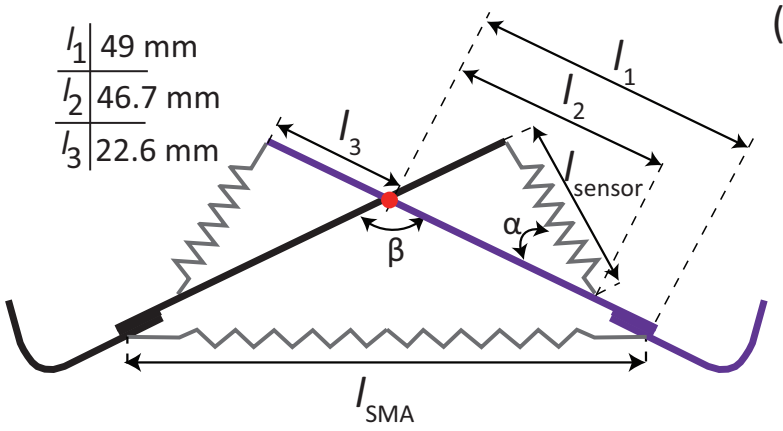

(b)

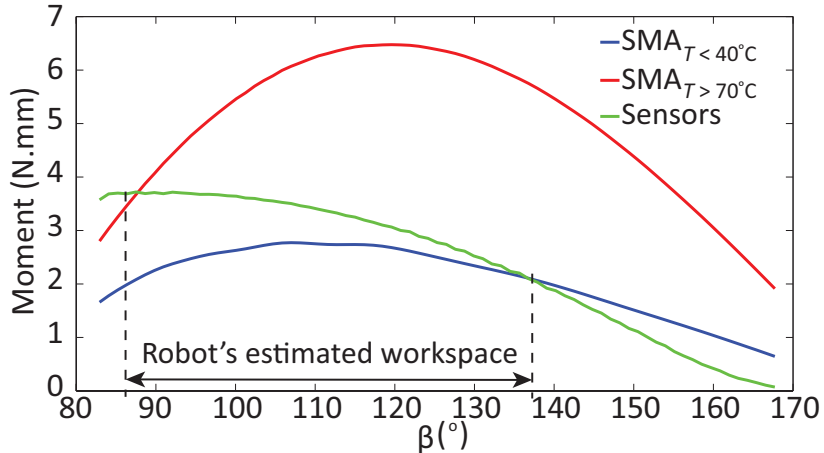

(c) 150

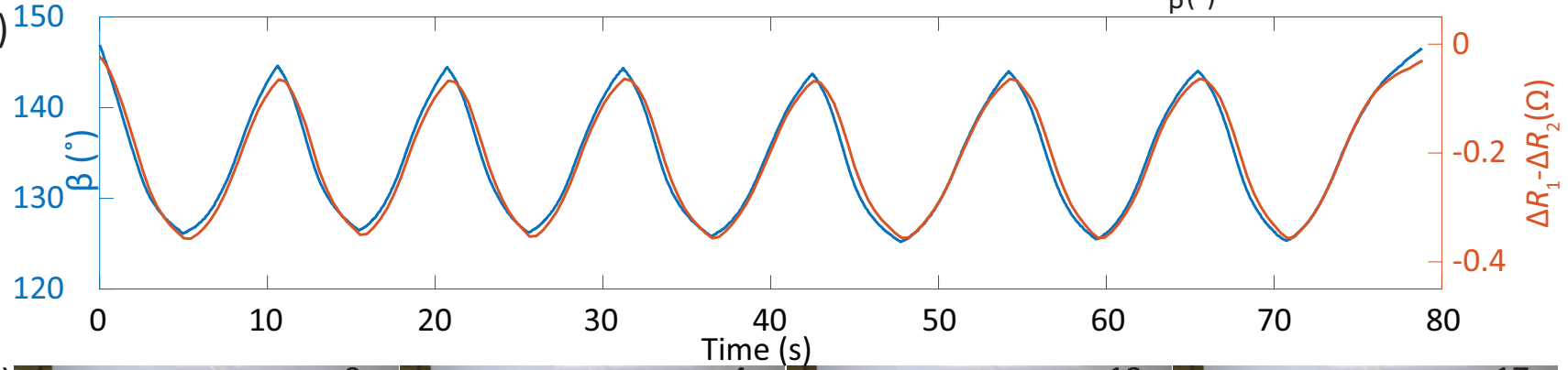

(d)

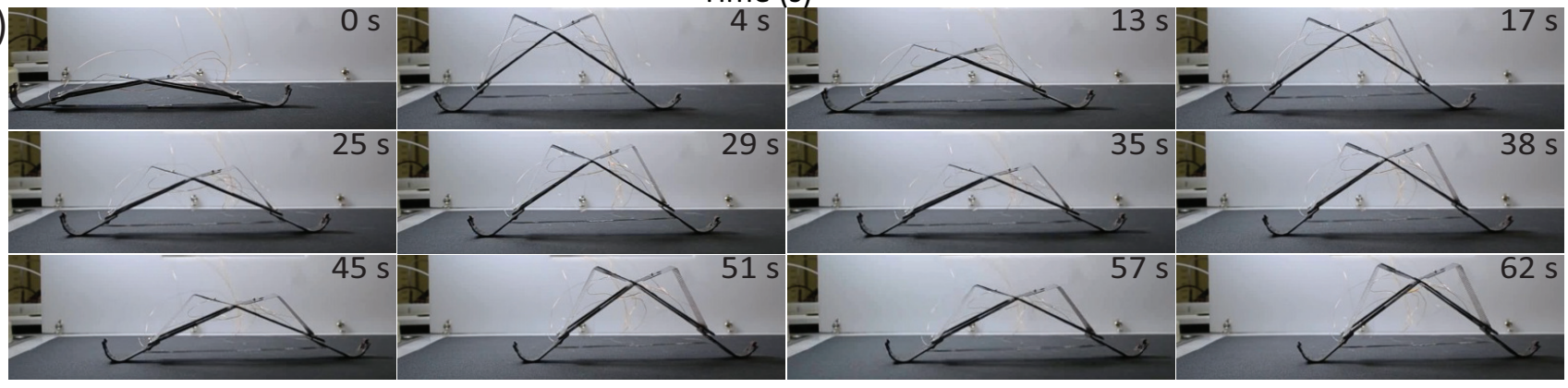

Fig. 10. Composite inchworm robot with functional Kirigami layers. (a) Kinematic design parameters. (b) Range of motion of the robot is estimated using characterization results (Fig. 7 and 9) and kinematic model. Due to the neglected joint stiffness, the actual workspace is slightly to the right of the estimated one. (c) Results of feedback controlled free motion in the horizontal plane. (d) Locomotion of the robot. In both free displacement and ground locomotion, SMA actuator draws $300 \mathrm{~mA}$ current at $10 \mathrm{~V}$ when activated.

the performance of an inchworm composite robot with three functional Kirigami layers: SMA Kirigami actuator, stretchable Kirigami sensor, and pop-up Kirigami scale. The layers in this robot are manufactured by laser micro-machining. After integration, the quasi-2D composite structure (Fig. 1) is folded into the final 3D shape of the robot; supplementary video. In the current prototype, the stationary joints are manually glued at their limits. This step could be automated in the future by integrating a heat shrinking layer. The axis of rotation and the joint limits, which determine the $2 \mathrm{D}$ to $3 \mathrm{D}$ transformation, are defined by the castellated pattern of the glass-fiber layers that make the main structure of the robot.

The foldable inchworm robot works based on the balance between the moments produced by the SMA actuator and stretchable sensors. Studying the kinematics of the inchworm robot allows us to minimize the number of required iterations for developing a well-balanced prototype. The equality of the moments applied to each leg of the inchworm robot by the actuator and sensors determine the equilibrium states of the robot at different SMA temperatures. These moments are calculated as:

$$
M_{\mathrm{SMA}}=F_{\mathrm{SMA}}\left(\text { Temp }, l_{\mathrm{SMA}}\right) l_{1} \cos (\beta / 2)
$$

$$
M_{\text {sensor }}=2 F_{\text {sensor }}\left(l_{\text {sensor }}\right) l_{2} \sin \alpha
$$

In (8) and (9), $l_{1}, l_{2}$, and $l_{3}$ are the geometrical design parameters and $\beta$ is the kinematic variable; introduced in Fig. 10a. $F_{\mathrm{SMA}}\left(T e m p, l_{\mathrm{SMA}}\right)$ in (8) is the function that evaluates the tensile force in the SMA actuator based on characterization results of Fig. 9; with actuator's length $\left(l_{\mathrm{SMA}}\right)$ and temperature (Temp) as inputs. Similarly, $F_{\text {sensor }}\left(l_{\text {sensor }}\right)$ in (9) evaluates the tensile force in the sensor based on the characterization results of Fig. 7; with its length $\left(l_{\text {sensor }}\right)$ as input. The length of the SMA actuator and the sensors are calculated as:

$$
\begin{gathered}
l_{\mathrm{SMA}}=2 l_{1} \sin \beta / 2 \\
l_{\text {sensor }}=\sqrt{l_{2}{ }^{2}+l_{3}{ }^{2}-2 l_{2} l_{3} \cos (\pi-\beta)}
\end{gathered}
$$

Using the kinematics of the robot, Equations (8)-(11) and the scaling laws (to navigate the design space of the Kirigami elements), the design of the inchworm and its components were refined in a few steps to achieve the desired range of motion as presented in Fig. 10b. We should mention that due to the neglected joint stiffness, the actual workspace is expected to be slightly different. 
Fig. 10c presents the results of feedback-controlled motion of the inchworm robot between two desired set points in free displacement (supplementary video). In this experiment, we estimated robot's state using measurements of two Kirigami sensors with opposite sensitivities. Combining the measurements of these two sensors provides a reliable estimation of the robot's state for achieving the repeated motion presented in Fig. 10c. Similar feedback controlled motion between two set points is used in the locomotion of the robot; Fig. 10d. The locomotion of the inchworm in the desired direction confirms the effectiveness of the scales in producing directional friction at the contact point with the ground. The inchworm composite robot illustrates the ease of integration of multiple functional Kirigami layers into a centimeter-scale robot using layer-bylayer manufacturing process.

\section{CONCLUSION}

In this research, we introduced a family of functional layers for meso-scale robots based on Kirigami patterns. Quasi2D Kirigami elements are manufactured with high precision (with 10s of micrometers features) using similar technologies previously utilized in the fabrication of composite robots, including laser micro-machining and engraving. Kirigami elements can assume different roles depending on the mechanical, thermal and electrical properties of the material. We presented three possible functions of Kirigami layers: SMA Kirigami actuators, stretchable Kirigami sensors and pop-up Kirigami scales. We integrated these functional Kirigami layers into an inchworm robot to illustrate their compatibility with the layerby-layer manufacturing process of composite foldable robots.

The multiple design parameters of Kirigami patterns provide a wide design space with the potential to meet requirements of different robotic applications. To navigate this design space, we studied the mechanical behavior of Kirigami layers and presented a model for the bending deformation of their comprising beams. We utilized this model to design actuator and return springs (also acting as stretchable sensors) with wellbalanced moments to drive the inchworm robot. The model's prediction for the twist angle was also used to design popup scales and to determine the required pre-stretch to reach a desired twist angle. This study provides a foundation to develop diverse functional Kirigami layers for application in composite robots with various requirements.

\section{REFERENCES}

[1] R. J. Wood, S. Avadhanula, R. Sahai, E. Steltz, and R. S. Fearing, "Microrobot Design Using Fiber Reinforced Composites," J. Mech. Des. vol. 130, 2008, Art. no. 052304.

[2] J. P. Whitney, P. S Sreetharan, K. Y. Ma, and R.J. Wood, "Pop-up book MEMS," J. Micromech. Microeng, vol 21(11), 2011, Art. no. 115021.

[3] A. M. Mehta et al., "Scripted Printable Quadrotor: Rapid Design and Fabrication of a Folded MAV," In: Inaba M., Corke P. (eds) Robotics Research. Springer Tracts in Advanced Robotics, vol 114. Springer, Cham.

[4] K. Jayaram and R. J. Full, "Cockroaches traverse crevices, crawl rapidly in confined spaces, and inspire a soft, legged robot," PNAS, vol. 113, no. 8, pp. E950- E957, 2016. In: Inaba M., Corke P. (eds) Robotics Research. Springer Tracts in Advanced Robotics, vol 114. Springer, Cham. Sci. Rep., vol. 8, 2018, Art. no. 3378.

[5] M. Noh, S. Kim, S. An, J. Koh and K. Cho, "Flea-Inspired Catapult Mechanism for Miniature Jumping Robots,' IEEE Trans.Robot., vol. 28, no, 5, pp. 1007-1018, 2012.
[6] M. E. W. Nisser, S. M. Felton, M. T. Tolley, M. Rubenstein and R. J. Wood, "Feedback-controlled self-folding of autonomous robot collectives," in Proc. IEEE Int. Conf. Robot. Autom., 2016, pp. 2541261.

[7] Z. Zhakypov, K. Mori, K, Hosoda, and J. Paik, "Designing minimal and scalable insect-inspired multi-locomotion millirobots," Nat. 571, 2019, pp. 381-386.

[8] M. Salerno, A. Firouzeh, J. Paik, "A Low Profile Electromagnetic Actuator Design and Model for an Origami Parallel Platform," ASME J. Mech. Robot. vol 9(4), 2017, Art. no. 041005.

[9] Z. Zhakypov, M. Falahi, M. Shah and J. Paik, "The design and control of the multi-modal locomotion origami robot, Tribot," in Proc. IEEE Int. Conf. Int. Robot. Sys., 2015, pp. 4349-4355.

[10] S. M. Felton, M. T. Tolley, C. D. Onal, D. Rus, and R. J. Wood, "Robot self-assembly by folding: A printed inchworm robot,' in Proc. IEEE Int. Conf. Robot. Autom., 2013 pp. 277-282.

[11] J. Koh and K. Cho, "Omega-Shaped Inchworm-Inspired Crawling Robot With Large-Index-and-Pitch (LIP) SMA Spring Actuators," IEEE/ASME Trans. Mechatronics, vol. 18, no, 2, pp. 419-429, 2013.

[12] J. Koh and K. Cho, "Omegabot: Crawling robot inspired by Ascotis Selenaria," in Proc. IEEE Int. Conf. Robot. Autom., 2010, pp. 109-114.

[13] S. Felton, M. Tolley, E. Demaine, D. Rus, and R. Wood, "A method for building self-folding machines," Science, vol. 345, pp. 644-646, 2014.

[14] D.-G. Hwang and M. D. Bartlett, "Tunable Mechanical Metamaterials through Hybrid Kirigami Structures," Sci. Rep., vol. 8, 2018, Art. no. 3378.

[15] Y. Zhang et al., "Printing, folding and assembly methods for forming 3D mesostructures in advanced materials," Nature Reviews Mat., vol. 2, 2017, Art. no. 17019.

[16] L. Tian et al.,'Flexible and Stretchable $3 \omega$ Sensors for Thermal Characterization of Human Skin," Adv. Funct. Mater., vol. 27, 2017, Art. no. 1701282.

[17] Y. Su et al.,'In-Plane Deformation Mechanics for Highly Stretchable Electronics," Adv. Mater., vol. 29, 2017, Art. no. 1604989.

[18] Y. Morikawa et al., "Ultrastretchable Kirigami Bioprobes," Adv. Healthcare Mater., vol. 7, 2017, Art. no. 1701100.

[19] N. Vachicouras, C. M. Tringides, P. B. Campiche, and S. P. Lacour, "Engineering reversible elasticity in ductile and brittle thin films supported by a plastic foil," Extreme Mech. Lett., vol. 15, pp. 63-69, 2017.

[20] L. Yin et al., "From All-Printed 2D Patterns to Free-Standing 3D Structures: Controlled Buckling and Selective Bonding," Adv. Mater. Tech., vol. 3, 2018, Art. no. 1800013.

[21] C. Wu, X. Wang, L. Lin, H. Guo, and Z. L. Wang, "Paper-Based Triboelectric Nanogenerators Made of Stretchable Interlocking Kirigami Patterns," ACS Nano, vol. 10, pp. 4652-4659, 2016.

[22] A. Firouzeh and J. Paik, "The Design and Modeling of a Novel Resistive Stretch Sensor With Tunable Sensitivity," IEEE Sens. J., vol. 15, pp. 6390-6398, 2015.

[23] A. Firouzeh and J. Paik, "Soft actuation and sensing towards robotassisted facial rehabilitation,' in Proc. IEEE Int. Conf. Int. Robot. Sys., 2017, pp. 306-313.

[24] H. Lee, K. Lee, K. Jang, and S. Ahn, "Shape Memory Alloy-Based Microscale Bending Actuator Fabricated by a Focused Ion Beam Chemical Vapor Deposition (FIB-CVD) Gap-Filling Process," Int. J. Precis. Eng. Man., 2019.

[25] Y. Tang et al., "Programmable Kiri-Kirigami Metamaterials," Adv. Mater., vol. 29, 2017, Art. no. 1604262.

[26] A. Lamoureux, K. Lee, M. Shlian, S. R. Forrest, and M. Shtein, 'Dynamic kirigami structures for integrated solar tracking," Nat. Commun., vol. 6,2015 , Art. no. 8092 .

[27] W. Wang et al., "Kirigami/Origami-Based Soft Deployable Reflector for Optical Beam Steering," Adv. Funct. Mater., vol. 27, 2017, Art. no. 1604214.

[28] A. Rafsanjani, Y. Zhang, B. Liu, S. M. Rubinstein, and K. Bertoldi, "Kirigami skins make a simple soft actuator crawl," Sci. Robot., vol. 3, no. 15, 2018, Art. 7555.

[29] J. K. Paik, R. K. Kramer and R. J. Wood, "Stretchable circuits and sensors for robotic origami," in Proc. IEEE Int. Conf. Int. Robot. Sys., 2011, pp. 414-420.

[30] M. Isobe and K. Okumura, "Initial rigid response and softening transition of highly stretchable kirigami sheet materials," Sci. Rep., vol. 6 , 2016, Art. no. 24758. 